\title{
Daria Tataj*
}

\section{INNOVACIÓN Y EMPRENDIMIENTO: UN MODELO DE CRECIMIENTO PARA ESPAÑA MÁS ALLÁ DE LA CRISIS**}

En este artículo nos centramos en analizar el modelo de crecimiento del Network Thinking, que permite obtener conocimiento útil tanto a ciudades como a regiones en el rediseño de sus propias políticas y estrategias de desarrollo y crecimiento económico. Dado que no existe un único modelo eficaz para todos los casos, el marco de crecimiento del Network Thinking ayuda a explicar cómo se genera innovación y emprendimiento en el contexto especifico de un ecosistema local al alinear a todos los agentes, las organizaciones y las instituciones clave, y en qué medida este ecosistema está conectado (o no) con las economías globales, en red e impulsadas por el conocimiento. El paradigma del Network Thinking constituye una herramienta analítica de gran utilidad para entender por qué determinados entornos favorecen la innovación más que otros y qué medidas puede tomar una ciudad o una región para acelerar el proceso de creación y captura del valor, definido por recursos clave como el conocimiento, el talento y el capital.

Palabras clave: innovación, emprendimiento, ciudad, región, política, crecimiento, Network Thinking. Clasificación JEL: L26, O30, O38, O40.

\section{Introducción}

Cada país y cada región del mundo adoptan el emprendimiento impulsado por la innovación de manera distinta. En función de las condiciones históricas, el contexto geopolítico, el acceso a la financiación y la cultura, los actores de un determinado ecosistema —nacional, regional o local- integran, colaboran

\footnotetext{
* Fundadora y CEO de Tataj Innovation. Asesora de alto nivel del Comisario para Investigación e Innovación de la UE, Carlos Moedas.

** Traducido del original Innovation and Entrepreneurship: A Growth Model for Spain Beyond the Crisis, por Armando Valdés Aumente.
}

(o no) y aprovechan sus conocimientos, tecnología, capital humano y base industrial de una manera específica para impulsar el crecimiento económico y la creación de empleo. Sin embargo, tanto los dirigentes políticos como los líderes empresariales aún toman Silicon Valley como punto de referencia y tratan de replicar el modelo económico basado en la innovación que dio lugar a este hito tecnológico. Pero seamos claros. Si bien es poco probable que se pueda replicar el modelo de Silicon Valley, la innovación y el emprendimiento suponen el único camino para que las 
ciudades posindustriales, tanto grandes como pequeñas, impulsen el crecimiento, creen empleos locales y eludan la trampa de convertirse en «lugares que ya no importan». Esta teoría resulta más cierta que nunca en la nueva era de la industria 4.0, la inteligencia artificial, las blockchain y los modelos de negocio basados en la economía colaborativa.

España, con su rico patrimonio industrial y con los lazos culturales que mantiene con el mundo hispano, su estabilidad política y económica garantizada por su pertenencia a la Unión Europea, y vibrantes ciudades creativas como Barcelona, Madrid, Málaga y Bilbao, está bien posicionada para convertirse en una de las principales economías mundiales en términos de innovación.

Sin embargo, la crisis financiera global acaecida entre 2008 y 2010 tuvo un efecto realmente perjudicial sobre la economía española y socavó el bienestar social y la solidaridad intergeneracional. Durante la última década, el país no fue únicamente una de las sociedades más perjudicadas, sino también una de las últimas economías en empezar (lentamente) a recuperarse de la crisis, y solo ahora está alcanzando los niveles previos de PIB. A lo largo de estos diez años de lucha, la crisis financiera obligó a las instituciones de crédito a restringir de manera drástica la concesión de financiación a empresas y hogares. Sin apoyo financiero, las empresas redujeron la inversión, recortaron los salarios y realizaron despidos, generando malestar social y provocando el surgimiento de partidos políticos radicales (Castells, 2017).

Las medidas de austeridad aplicadas para devolver las economías en crisis a la senda del crecimiento han motivado la congelación de los presupuestos públicos, lo que ha desembocado en una abrupta caída del consumo y en una reducción de la demanda doméstica, que representa en torno a tres cuartas partes del crecimiento del PIB de la economía española. La Unión Europea acudió en ayuda del país. Así, se rescataron los bancos españoles con dinero de los contribuyentes europeos y se estimuló la demanda mediante la inversión pública en infraestructuras y subvenciones sociales. Dado que el nivel del endeudamiento del Gobierno español y los consumidores individuales era ya elevado, la deuda pública experimentó un acusado repunte y los mercados financieros elevaron la prima de riesgo y los tipos de interés de dicha deuda pública hasta alcanzar niveles insostenibles. La Comisión Europea, con la ayuda del Fondo Monetario Internacional, intervino para rescatar a España a cambio de imponer estrictas políticas que ayudasen a lidiar con problemas de solvencia a corto plazo. Sin embargo, estas agravaron el estancamiento de las actividades económicas y agudizaron la tendencia a la baja de la inversión extranjera directa.

Un escenario similar al ocurrido en España se pudo ver en Portugal, Italia y Grecia, y en distinta medida en todos los países de la Unión Europea que sufrieron la recesión económica, con una notable excepción: Polonia. Merece la pena analizar el caso de este último país para extraer enseñanzas de su modelo de crecimiento.

\section{Aprender de los mercados emergentes: las enseñanzas de Polonia}

Con 38.000.000 de habitantes, Polonia es uno de los Estados miembros más extensos de la UE y la mayor economía de los mercados emergentes de Europa Central y del Este. En su informe titulado Insights for Poland. Lesson from Poland, el Banco Mundial (2018) afirma:

«El ascenso económico de Polonia es notable. Después de la transición desde el comunismo, el PIB per cápita de Polonia experimentó un crecimiento rápido y estable, con un promedio aproximado del 4 por 100 anual. El país pasó de un nivel de renta media a alta en menos de 15 años. La economía se expandió rápidamente debido en dos terceras partes al esfuerzo (inversión) y en una a la inspiración (innovación). Y la prosperidad fue generalizada. El crecimiento de los ingresos y los puestos de trabajo tuvo un alcance amplio y las regiones rezagadas han venido recuperando 
terreno. Durante el tiempo en que se materializaron estos cambios, el coeficiente de desigualdad en los ingresos (Gini) no se incrementó. Polonia tiene uno de los coeficientes de Gini más bajos entre los países que han pasado a ser de renta alta desde 2000 (los «nuevos países de renta alta», HIC por sus siglas en inglés). Asimismo, presenta una de las menores diferencias regionales internas en materia de PIB per cápita».

No existe una razón única que explique por qué este país ha tenido tanto éxito en términos de crecimiento y desarrollo, y por qué ha demostrado una resistencia tan firme ante la crisis económica mundial. De hecho, Polonia, que llevaba siendo miembro de la UE solo cuatro años cuando la crisis comenzó, logró mantener un crecimiento positivo del PIB durante toda ella, alcanzando el 4,5 por 100 en 2011 (Eurostat).

Las razones que han propiciado esta situación son múltiples y de efecto sinérgico. Sin duda, entre ellas se incluye la entrada de fondos de preadhesión y estructurales de la Unión Europea percibidos no solo como un flujo de euros, sino como "dinero inteligente» que trae consigo la transferencia de experiencia y conocimientos. Un ejemplo puede ser el sector financiero. $Y$ es bien sabido que uno saludable constituye el pilar de cualquier economía de libre mercado. Las instituciones financieras de libre mercado de Polonia se han creado desde cero durante los últimos 30 años hasta convertir en la actualidad al sector bancario polaco en uno de los más avanzados del mundo desde el punto de vista tecnológico. Especialmente importante es el sector de los servicios financieros para pymes, que impulsa el crecimiento y la innovación de esta economía emprendedora. El know-how entró al país con la inversión extranjera directa y también con programas como el instrumento de financiación para las pymes de la UE/BERD, uno de los planes de financiación de preadhesión destinados a impulsar la creación de un sector de las pymes sólido. Partiendo de la falta de capacidad para financiar el crecimiento de las pequeñas y medianas empresas, las instituciones bancarias más grandes, como PKO BP, ING Bank Sląski o BZ
WBK, acometieron una transformación desde dentro. En el caso de las dos últimas, dicha transformación vino propiciada por la transferencia de conocimientos aportada por los inversores extranjeros. En el caso de la primera, PKO BP, una antigua entidad pública, la ayuda de la asistencia técnica de la UE resultó fundamental para modificar los procedimientos y los procesos de concesión de créditos a pymes y para institucionalizar un cambio que afectó al 25 por 100 de las cuentas bancarias de las empresas de pequeño y mediano tamaño de Polonia.

Sin embargo, la afluencia de fondos de la UE, con o sin asistencia técnica, no explica la resistencia de la economía y su crecimiento a largo plazo.

La educación fue indudablemente uno de los principales vectores que impulsaron el éxito del país. Los resultados de la prueba PISA de la OCDE ponen de relieve que Polonia ha escalado desde una posición inferior a la media de la OCDE hasta situarse en el cuarto puesto en Europa y el décimo segundo a nivel mundial en 2014. Cada año unos 400.000 graduados acceden al mercado de trabajo y una de las principales características que los hacen destacar es su mentalidad emprendedora.

Su cultura empresarial, que aúna creatividad, trabajo duro, inconformismo y el deseo de transformar el país, se basó en alcanzar el sueño compartido de aspirar a un futuro mejor que propulsase la transformación de Polonia después de los Acuerdos de la Mesa Redonda de 1989 entre los dirigentes del sindicato Solidaridad y el Gobierno. Posteriormente, jugó un papel fundamental el esfuerzo diario de muchas personas de la clase trabajadora para adoptar este cambio histórico y desarrollar el capitalismo en la práctica, estableciendo sus pequeños negocios, a menudo familiares, participando en el comercio y satisfaciendo las necesidades de clientes de recursos limitados, a menudo pertenecientes a la «base de la pirámide». La transformación, el ritmo sostenible, el rumbo y los valores subyacentes de Polonia constituyen un modelo alentador de crecimiento y desarrollo social que se 
debe compartir y del que debemos aprender. A modo de advertencia, se debe tener en cuenta que la innovación en los mercados emergentes responde a distintos factores, a menudo poco o nada tecnológicos, pero aun así impulsores de un valor altamente disruptivo. Si bien en el caso de los mercados emergentes, la escasa base científica y tecnológica general se ve compensada con la innovación en materia de modelo organizativo y empresarial, en los mercados maduros, como es el caso de España, la ventaja real procede de la capacidad para interconectar la investigación, la educación, la innovación y el espíritu emprendedor.

3. Innovación y emprendimiento: un modelo de crecimiento para Europa más allá de la crisis

La teoría económica y la experiencia histórica reciente muestran que el crecimiento de la productividad se debe en gran parte a la innovación y el espíritu emprendedor. La innovación crea riqueza a partir de la sinergia que resulta de combinar los factores de producción a lo largo del proceso productivo (crecimiento de la productividad multifactorial en términos econométricos tradicionales). Por su parte, el espíritu emprendedor implica que existen actores económicos que organizar y financiar, y nuevos productos y procesos que sacar al mercado.

Sin embargo, saber que la innovación y el espíritu emprendedor son las fuentes de productividad y competitividad no responde a la pregunta principal: ¿cómo se generan la innovación y el espíritu emprendedor en nuestras economías globales, conectadas y basadas en el conocimiento, y en el contexto específico de países europeos como España? Y, ¿por qué determinados entornos favorecen la innovación? ¿qué acelera el proceso de creación y captación de valor? ¿por qué algunas ciudades atraen recursos tales como el conocimiento, el talento y el capital mientras que otras se convierten en «lugares que ya no importan»?

La respuesta radica en la teoría y las prácticas emergentes de innovación de la economía impulsada por el conocimiento.
Cuando entendemos cómo se crea valor en nuestro entorno conectado, tanto en mercados maduros como emergentes, la innovación aparece como un sistema de aprendizaje omnipresente que se perpetúa. Con ello no nos referimos a la educación, sino a un aprendizaje colectivo basado en intercambiar información en tiempo real y compartir conocimientos. En la era de la información, la educación formal y sus instituciones coexisten en paralelo a múltiples entornos formativos que emergen en función de las necesidades puntuales tanto en el mundo real, como es el caso de espacios de creadores, el movimiento Fab Lab, las reuniones meet-ups o hackathons, como en el mundo digital, donde las personas aprenden «reuniéndose, pasando el rato y curioseando» en Internet. La innovación e innovar se convierten en un proceso en desarrollo de aprendizaje abierto, distribuido y colectivo, y representan básicamente la capacidad de innovar en un mundo en constante transformación.

La idea principal del modelo de crecimiento del Network Thinking (pensar en red) se basa en el marco conceptual desarrollado en la publicación «Innovation and Entrepreneurship. A Growth Model for Europe Beyond the Crisis» por la autora del presente artículo. Incluye cuatro pilares esenciales para el éxito operativo de las redes de innovación en la economía del conocimiento: la investigación, la educación, la innovación y el espíritu emprendedor —aplicados a las estrategias de negocio y la formulación de políticas-. El paradigma del crecimiento del Network Thinking se condensa en un modelo sencillo. Lo que resulta más complejo es darse cuenta de que estos cuatro pilares determinan tanto a las instituciones tradicionales, donde el conocimiento se ha producido, difundido y comercializado, como a los propios procesos, dado que en la economía digital estas actividades se realizan más allá de los límites institucionales, en nuevos paradigmas de ciencia e innovación abiertas y entornos educativos híbridos mixtos (Figura 1).

La función clave del emprendimiento, que se sitúa en el centro de la red de innovación y está compuesta 


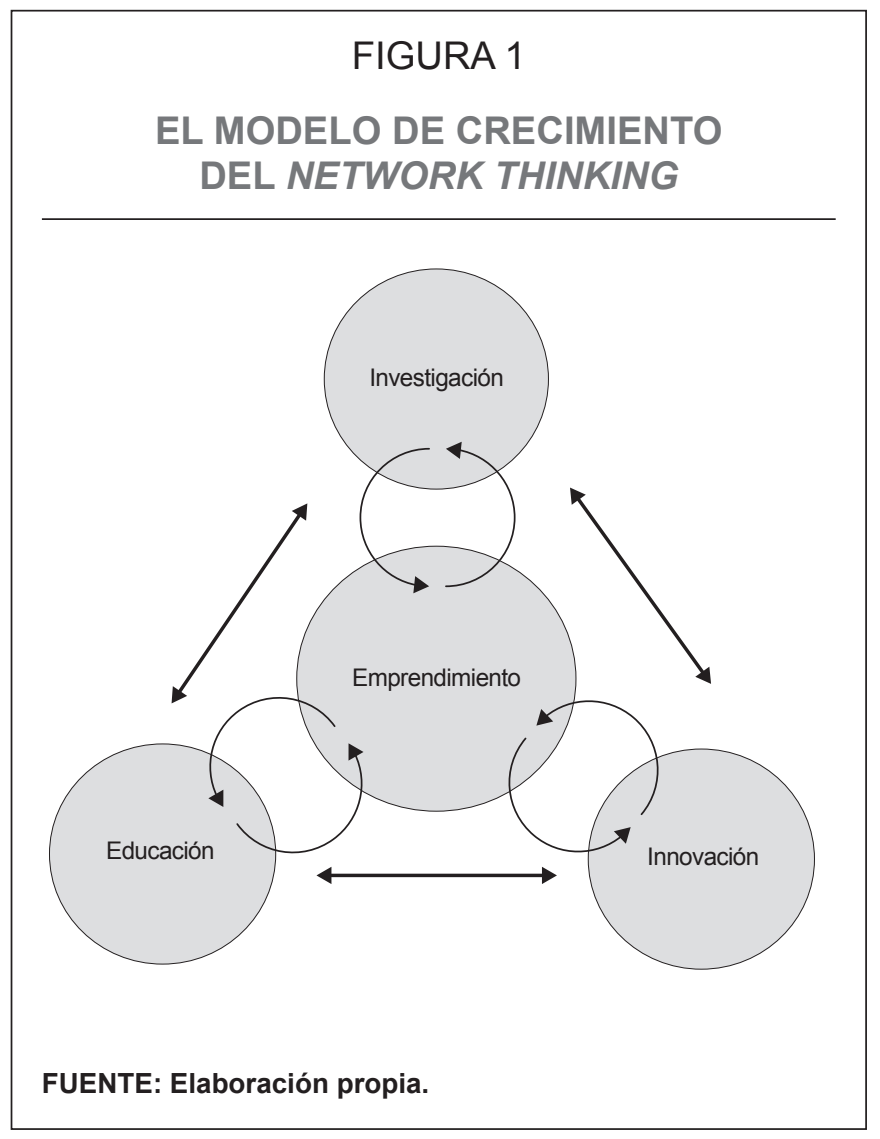

por la investigación, la educación y la innovación, y a la que se alude en la formulación de políticas europeas como el «triángulo del conocimiento», apunta al principal elemento ausente para la creación de valor, que es el fomento de la capacidad colectiva de desarrollar nuevos productos y procesos, y lanzarlos a los mercados y a la sociedad de manera oportuna y puntual. Las pruebas demuestran que se puede reducir la brecha entre la innovación y los mercados a través de un conjunto diverso de entidades empresariales que comienzan como pequeñas empresas innovadoras hasta convertirse en grandes multinacionales, pasando por organizaciones sin ánimo de lucro, así como instituciones públicas, incluidas las universidades y las personas físicas individuales: profesionales autónomos creativos y emprendedores micromultinacionales.
En el modelo de crecimiento basado en el Network Thinking, no se establece una jerarquía entre los distintos entornos donde se produce la innovación. En los mercados emergentes, la «torre de marfil» del mundo académico no es más eficaz que otros contextos, laboratorios de empresas o de ciudades, como plataforma de innovación donde los participantes observan una transición desde las culturas transaccionales impulsadas por los intercambios y la lógica del «juego de suma cero» hasta otras culturas organizativas emergentes basadas en la confianza, las relaciones y la colaboración, habituales de entornos innovadores.

En estos entornos, ejemplificados por Living Labs o espacios creativos, los conocimientos codificados se transmiten por medios tangibles, a menudo a través de acuerdos formales de colaboración y regímenes de propiedad intelectual, mientras que los no codificados se transmiten a través de personas físicas y se difunden únicamente en función de la decisión de estas de compartirlos o no. Los entornos de innovación abierta son producto de la transición de culturas transaccionales a otras colaborativas basadas en la confianza. Y estos flujos de conocimientos resultan de vital importancia para que se produzca la innovación, ya que brindan una capacidad única para formular los conocimientos codificados. De esta forma, se impulsa el proceso de recombinación, reconexión y reformulación para un contexto cambiante. En resumen, le da un nuevo significado; un significado que crea valor.

La dinámica de estos flujos continuos de conocimientos facilita una cultura del cambio, esto es, una cultura de aprendizaje constante. El sistema de intercambio de conocimientos, omnipresente y que se perpetúa a sí mismo, se convierte en uno de los ingredientes principales de la innovación en un mundo sujeto a incesantes cambios.

Lo que mantiene unidas a las redes de innovación transnacional, transinstitucional, transectorial e interdisciplinaria es, por una parte, la amplia variedad de modelos disponibles para estructurar colaboraciones, vínculos y alianzas contractuales formales $\mathrm{y}$, por otra, 
la capacidad de los miembros de la red para crear valor de manera colectiva. Esta última es la conexión que aglutina a la red desde el punto de vista operativo. Un plan de negocio o un proyecto de innovación social comienza por identificar los mecanismos de creación de valor ejemplificados por diversos modelos de negocio en red, en lo que representa un nuevo paradigma de empresa en red. La empresa en red se define como una unidad, con o sin estructura jurídica, de duración limitada y organizada con el fin de lograr un objetivo concreto, para lo cual diversos actores asignan recursos y crean un proyecto.

El emprendimiento constituye el componente integrador que mantiene unido el «triángulo del conocimiento». Resulta fundamental para integrar redes en diversos entornos donde se producen los componentes del proceso de innovación. Y actúa como un catalizador para la creación de valor, no solo mediante la integración de la red, sino también a través de la creación de sinergias entre sus componentes.

Por lo tanto, lo que inicialmente se presentó como el triángulo del conocimiento se reformula como una red multidimensional, en la cual, tres componentes del proceso -investigación, educación e innovación- concurren simultáneamente en diversos entornos en una interacción de efecto sinérgico. Esto marca un cambio conceptual que se aleja de la interpretación de la innovación como una forma de colaboración bilateral o multilateral entre actores del ámbito académico e industrial, y con diversos grados de participación del Estado.

El nuevo paradigma de Network Thinking concibe el proceso de innovación de la producción, difusión y comercialización o aplicación del conocimiento dentro de un contexto dinámico, abierto y horizontal donde fluyen diferentes tipos de conocimientos impulsados por el talento emprendedor y el capital empresarial. El triángulo del conocimiento se convierte en un constructo multidimensional, dinámico y emergente «en desarrollo». Y como resultado del análisis, los componentes del triángulo del conocimiento se caracterizan por una transición, que va desde la investigación basada en la propiedad intelectual hasta la innovación abierta; desde la enseñanza universitaria hasta entornos educativos con un sólido componente de aprendizaje entre pares; desde la innovación en el marco de la industria hasta aquella que se produce en distintos tipos de redes sociales, creativas y de procesos.

Estos entornos propicios para la innovación están interrelacionados y mantienen un vínculo de dependencia mutua en el contexto de la red global de las redes de innovación, que impone su lógica, dinámica y estructuras en las prácticas de gestión y los modelos de negocio. Lo que llevamos tiempo observando es que el conocimiento, el talento y el capital fluyen libremente a través de las fronteras y que estos recursos se acumulan en determinadas zonas metropolitanas. El proceso de agrupamiento sectorial data de las primeras fases de la revolución industrial.

Sin embargo, los modelos de negocio emergentes de empresas participantes en redes globales y locales alteran los métodos de creación de valor. Ciertas regiones o ciudades emergen como grandes nodos y obtienen acceso a fuentes globales de recursos. Se convierten en imanes que atraen conocimientos profundos, emprendedores y capital inteligente. Algo que se observa en determinados casos, y que es más propicio de cara al éxito, es el cambio del triángulo de conocimiento, que pasa de beber de culturas transaccionales impulsadas por los intercambios y la lógica del «juego de suma cero», a cimentarse en culturas organizativas basadas en la confianza, las relaciones y colaboración, habituales de entornos innovadores.

El análisis de la estructura y las modalidades de los diferentes tipos de redes de innovación pone de relieve cómo diversas características, factores y mecanismos provocan la evolución de dichas redes. El proceso de evolución de los nodos de red nos brinda una explicación de las razones por las cuales algunos de estos nodos cambian mediante la atracción de flujos de conocimientos, talento y capital. La acumulación de estos recursos modifica el estado de los nodos en la arquitectura global de las redes de innovación. Se 
convierten en centros de innovación. La estructura, la arquitectura local y global y la dinámica de los flujos entre los nodos y la periferia ponen al descubierto los factores subyacentes y los catalizadores que aceleran la velocidad de los flujos y la generación y la acumulación de riqueza.

El aspecto clave del modelo de crecimiento del Network Thinking es la forma en que se crea valor en las redes de innovación a través de diferentes tipos de modelos de negocio, entendiéndose estos como un sucedáneo de los modelos de creación de valor. El marco conceptual de una empresa de red permite comprender la singularidad de esta unidad organizativa dentro de una economía de red. Los emergentes modelos de negocio de la red de innovación mundial aprovechan este nuevo paradigma de empresa de red para entender e interpretar fenómenos como las redes sociales y las redes de innovación abierta, así como las redes de procesos y las redes de creación.

Por último, la contradicción fundamental entre los mecanismos de acumulación de conocimientos y riqueza dominantes en la sociedad en red y la dinámica de los flujos a través de las redes de innovación genera un nuevo mapa de desigualdad social, económica y tecnológica. Algunas regiones prosperan y se modernizan. Otras sufren la fuga de cerebros, el desempleo y la radicalización de la escena política. Este esquema analítico puede aplicarse tanto a escala macro en una región de un país, como a nivel micro en empresas.

Uno de los experimentos más atrevidos llevados a cabo en el seno de la Unión Europea ha sido la puesta en marcha del Instituto Europeo de Innovación y Tecnología (IET), creado en 2008 por la Comisión Europea, y sus comunidades de conocimiento e innovación $(\mathrm{CCl})$. Las tres primeras $\mathrm{CCl}$ se constituyeron en 2010 como respuesta estratégica y organizativa para abordar grandes desafíos sociales en materia de energía y clima y de la futura sociedad de la información y la comunicación. Estas se crearon para experimentar con nuevos modelos de crecimiento, que reúnen a grandes empresas, institutos de investigación y universidades en redes locales de innovación conectadas en redes paneuropeas que, en su conjunto, conforman la comunidad de CCI del IET. Las comunidades de conocimiento e innovación representan la aplicación experimental de los principios del Network Thinking al modelo del triángulo del conocimiento impulsado por el emprendimiento. Fueron creadas por el IET como asociaciones colaborativas con estructura jurídica y financiera entre la industria y el mundo académico.

Lo que podemos aprender de la trayectoria de la evolución de las $\mathrm{CCl}$ es que el modelo ha tenido un impacto indudable. Hasta la fecha, las $\mathrm{CCl}$ han constituido 600 startups que han creado más de 14.000 empleos y cuentan con una red de 4.000 graduados y miles de estudiantes en el sistema de educación empresarial, que conectan la ciencia y la tecnología con las habilidades, la perspectiva y las conexiones necesarias para construir nuevas empresas. El experimento implica un cambio multidimensional que adopta el emprendimiento como nexo entre los elementos del triángulo del conocimiento. En el contexto de la formulación de políticas, nuevas instituciones como el IET y sus $\mathrm{CCl}$ pueden incrementar considerablemente la probabilidad de éxito de las alianzas colaborativas entre el mundo universitario y la industria, y apoyan o lastran la transición estratégica hacia un nuevo tipo de institución académica, la innovación abierta y la creación de iniciativas empresariales de alto crecimiento respaldadas por fondos de capital de riesgo.

\section{4. ¿Qué aprendizajes podemos extraer del Tour d'Europe del RISE?}

Para el futuro de Europa, la capacidad para mantener un modelo socioeconómico basado, por una parte, en los principios de la economía de libre mercado y, por otra parte, en la opción política de los ciudadanos europeos en favor del Estado de bienestar, pasa por contar con las políticas adecuadas para apoyar el crecimiento de la productividad y las exportaciones como faros que muestran el camino hacia una nueva 
prosperidad más allá de la crisis económica en cada Estado miembro, entre ellos España.

Una de las causas principales de la crisis en la eurozona fue la integración artificial de economías con enormes diferencias en términos de productividad, cultura e instituciones, sin una unión fiscal y sin un sistema bancario regulado e integrado. Si bien la crisis indujo a que la UE sentase los cimientos de un sistema bancario y de una política fiscal comunes, aún queda por resolver la cuestión de cómo organizar todas las economías para que incrementen la productividad y la competitividad.

EI Tour d'Europa del RISE brinda una serie de respuestas para esta cuestión fundamental. RISE es el acrónimo del grupo Research and Innovation Staff Exchange del comisario europeo Carlos Moedas. La misión del grupo RISE consiste en proporcionar al comisario y a su gabinete apoyo directo para crear nuevos enfoques, marcos e instrumentos políticos. En 2015, el grupo publicó su libro The Future of Europe: Open Science, Open Innovation, Open to the World. El libro se basa en el manifiesto del comisario Moedas partiendo de las «tres oes» (Open Science, Open Innovation y Open to the World). Partiendo de estos tres componentes, el grupo RISE ha presentado sus reflexiones colectivas, contribuyendo de esta manera a reforzar los nuevos elementos del programa marco Horizonte Europa (2021-2027). Estos incluyen, entre otros, la adopción de un enfoque orientado a misiones determinadas y el Consejo Europeo de Innovación.

Desde el otoño de 2017 y hasta la finalización de 2018 , los miembros del RISE han visitado diversas capitales europeas para reunirse con expertos independientes y laboratorios de ideas, a fin de presentar su libro y comentar en este contexto las agendas nacionales de investigación e innovación, l+i. Esta gira ha sido diseñada en el marco de la iniciativa impulsada por el presidente de la UE, Jean-Claude Juncker, que apuesta por llevar a cabo un debate amplio y abierto sobre la futura dirección del proyecto europeo.

En octubre de 2017, después de la sesión de apertura del Parlamento Europeo y de las visitas realizadas a París y Estocolmo, los asesores del RISE celebraron una reunión con expertos españoles auspiciada por la Fundación Cotec. La visita a Madrid del Tour d'Europe se centró en el campo de la I+i, en general, y en el modelo de economía circular, en concreto, y en su función dentro de la política europea, en su conjunto. En la reunión se mantuvo un debate entre el grupo RISE y los 100 de Cotec, una red de importantes expertos independientes, entre los que se encontraban asesores políticos, economistas, académicos e intelectuales.

La reunión giró en torno a tres temas principales: i) la necesidad de cambiar la narrativa sobre la política de I+i de la UE; ii) el acercamiento a la economía circular como objetivo clave de la UE; y iii) las reformas estructurales que son necesarias para aportar un carácter más abierto al sistema de I+i en Europa.

En primer lugar, Europa necesita una nueva narrativa para el proyecto europeo en general, y el Espacio Europeo de Investigación, junto con la competitividad del mercado único, son algunos de los aspectos concretos que debe incorporar esta narrativa más amplia: «¿por qué Europa? ¿qué Europa?». En el primer caso, el postulado pasaba por distanciarse de las comparaciones con Estados Unidos y China, ya que la mayor parte de los europeos rechazan seguir los modelos políticos, sociales y económicos de estas dos naciones. La verdadera pregunta es: ¿qué preocupa a las nuevas generaciones europeas? ¿cuáles son sus sueños y valores? y ¿cómo interpretan en la práctica la preferencia europea por el Estado de bienestar, una sociedad abierta y una Europa soberana que beneficie a todos los europeos con inversión pública y un impacto real?

En segundo lugar, el debate hizo hincapié en la necesidad de acometer un cambio de paradigma para la $1+\mathrm{i}$, sustituyendo el enfoque de subvenciones públicas por otro que apueste por la inversión estratégica. En el lenguaje de los procedimientos administrativos, cuando se alude a ayudas se da por sobreentendido que se trata de subvenciones públicas. Parte de la nueva narrativa trata de alejarse de pensar en términos de «solicitar subvenciones», «obtener subvenciones» o 
«utilizar subvenciones» bajo la lógica implícita de que la subvención es un medio para impulsar los indicadores clave de rendimiento de cara a alcanzar la excelencia científica, esto es, generar investigación de calidad plasmada en la cantidad de publicaciones académicas y en el impacto por número de citas.

Se abre una perspectiva muy diferente si concebimos los programas marco como una inversión que debería generar un impacto equilibrado tanto en la sociedad como en la economía - y no solo en la investigación como un ámbito independiente- - Las I+i son factores clave de la economía y pueden crear una sociedad mejor, aportar soluciones para abordar los principales retos y ayudar a alcanzar los Objetivos del Milenio. En el debate se alzaron voces que reclamaban que la financiación pública en I+i podría y debería medirse más allá de indicadores científicos específicos e incluir, por ejemplo, las mejoras en la vida de los ciudadanos europeos o la creación de nuevos puestos de trabajo. Esta forma de pensar modifica el paradigma de lo que significa el presupuesto de $\mathrm{I}+\mathrm{i}$, tanto para los dirigentes políticos como para los investigadores. Se les reclama a ambos colectivos que rindan cuentas ante los ciudadanos y que el gasto en I+i no debería ponerse en contra de otras inversiones, como por ejemplo la inmigración o la agricultura sostenible, sino que deberían abordarse a través de la propia inversión en I+i. En resumen, la I+i puede aportar formas eficaces de mitigar los retos de aspectos tales como los movimientos migratorios o la contaminación vinculada a la actividad agrícola. Este nuevo enfoque, en el que la innovación se entiende como un proceso de aprendizaje, requiere la apuesta y el esfuerzo del conjunto de la sociedad para asimilar lo que la investigación le pueda ofrecer. Y para facilitar este proceso resulta primordial la comunicación entre las redes que conforman los ecosistemas de innovación.

En tercer lugar, el programa Horizonte 2020 introdujo el concepto de desafíos sociales, que puede articularse a través de los Objetivos del Milenio. Sin embargo, esta iniciativa no ha tenido el impacto transformador esperado. En consecuencia, el grupo RISE afirmó que los dirigentes políticos europeos encargados de la $1+\mathrm{i}$ deberían dar un paso adelante hacia una política orientada a misiones concretas, a fin de aprovechar al máximo las inversiones realizadas hasta la fecha, incrementando al mismo tiempo la rentabilidad de las nuevas. Por ejemplo, el cambio hacia una economía circular podría convertirse en una de estas misiones. De ser así, para impulsar este cambio, se debería contar con la participación de los ciudadanos en el diseño de nuevos productos, servicios y soluciones industriales. De cara a darles un uso más amplio, sería necesario coordinar y combinar la inversión pública con el crowdfunding, la $1+i$ con el progreso social o los cambios regulatorios $y$, ante todo, el ingente número de nuevas startups con modelos de crecimiento sostenible. Asimismo, existen otras muchas áreas donde la $1+i$ constituye un factor necesario pero insuficiente para mejorar las vidas de los ciudadanos de Europa. La lucha contra el cáncer es una misión potencial que, como cualquier otra, necesitará contar con un enfoque propio que combine un ecosistema coordinado de movilización de recursos con la organización de las actividades de innovación, tanto de arriba abajo como de abajo arriba.

En conclusión, la visita del Tour d'Europe de RISE a Madrid puso el foco sobre aspectos clave relativos a la redefinición de reformas estructurales en el ámbito de la l+i en el contexto de España. En este país, como en muchos otros Estados miembros, el vínculo entre conocimientos e innovación está roto, es decir, la inversión en I+i no se traduce en crecimiento. La excelencia como tal y por sí sola no crea empleos de calidad. Y si tuviéramos que elegir un único indicador simple del estado de la economía y la sociedad europeas, la creación de nuevos empleos de calidad en Europa podría ser el parámetro definitivo que nos proporcione una visión clara de la salud del proyecto europeo en línea con el contexto del presidente Juncker.

En tal caso, el proceso que está llevando a cabo el Espacio Europeo de la Investigación debería basarse en la apertura más que en la linealidad, y los «costes de la no Europa» en I+i podrían contraerse si los 
programas nacionales y comunitarios se diseñasen con el objetivo de generar sinergias. De cara a aprovechar estas sinergias potenciales, las universidades deben abrirse a incorporar innovación abierta y ciencia abierta a sus estrategias, prácticas y sistemas de incentivos. Es preciso contar con modelos que sirvan como ejemplo y, para ello, el Consejo Europeo de Investigación debe mostrar el rumbo a seguir para adoptar realmente prácticas de acceso abierto y datos abiertos. Igualmente, el Instituto Europeo de Innovación y Tecnología debería iniciar nuevos proyectos de colaboración abierta para aprender de las prácticas de sus comunidades de conocimiento e innovación cómo salvar la distancia entre los grandes retos sociales y las misiones concretas.

Por último, en la reunión se aludió a la necesidad de reforzar la cooperación entre las instituciones europeas y los organismos nacionales a fin de garantizar la eficacia de toda política en innovación. Asimismo, se hizo hincapié en la función de las universidades como agentes organizativos y en el nuevo reglamento para impulsar su apertura y facilitar la experimentación local de soluciones innovadoras, simplificando los instrumentos, desarrollando nuevas alianzas y formas más flexibles de agrupamiento de empresas, métodos diversificados y el aprovechamiento de sinergias arraigadas en la función del Estado a través de contrataciones públicas y el acceso a nuevos mercados sin los obstáculos de las normas sobre ayuda estatal. Al final, los expertos reclamaron que se identificase un método más eficaz de medición del impacto, lo cual supone una tarea complicada, dado que la política basada en hechos muestra que no deberíamos seguir los modelos políticos lineales/verticales de las pasadas décadas. No obstante, aún resulta confuso el camino que se debe seguir para progresar en este ámbito.

\section{Universidades emprendedoras y nuevos espacios de colaboración}

La visita de RISE a la Fundación Cotec supuso la culminación de las líneas de trabajo multidimensionales del grupo. Una de estas líneas está relacionada con las universidades emprendedoras y los nuevos espacios formativos emergentes y colaborativos, como espacios de creadores, Fab Labs, centros de coworking y todo tipo de aceleradores de transferencia de conocimientos, meet-ups, hackathons y jornadas de startups. En septiembre de 2017, en colaboración con la Universitat Politècnica de Catalunya-BarcelonaTech (UPC), el grupo RISE organizó una reunión política de alto nivel para abordar este tema y analizar métodos para facilitar que las universidades europeas establezcan nuevos tipos de asociaciones en línea con las políticas de las «tres oes» del comisario Moedas, esto es, innovación abierta, ciencia abierta y apertura al mundo.

Esta reflexión se enmarcó dentro del concepto de universidad emprendedora, es decir, una universidad que adopte la «tercera misión»; un enfoque estratégico como miembro del ecosistema local que fortalezca las políticas públicas y las prácticas de negocio a fin de impulsar el desarrollo económico, en este caso, a través del emprendimiento. Las tres cuestiones que se sometieron a debate fueron las siguientes:

- En este enfoque centrado en las personas, que esgrime RISE como uno de los ejes principales de la transformación para el próximo programa marco de Investigación e Innovación de la UE, ¿cómo pueden las universidades capacitar, incentivar y apoyar tanto a empresarios como a emprendedores sociales?

- ¿Qué se necesita para liderar el cambio de una institución académica tradicional a una universidad abierta de la era digital?

- ¿Cómo se puede aplicar un enfoque ecosistémico al desarrollo de nuevos tipos de colaboración entre universidades, empresas, Gobiernos, ciudades y la sociedad en su conjunto?

El tema de esta reunión del grupo RISE proviene de la idea de converger, por un lado, los enfoques verticales (de arriba abajo y de abajo arriba) de un dirigente político sobre cómo fomentar un ecosistema de innovación abierta en toda Europa para crear un entorno propicio para la prosperidad económica y el bienestar social y, 
por otro lado, las tareas estratégicas de un líder universitario que se enfrenta al reto de identificar un método para reforzar el modelo de transferencia de conocimientos de una universidad politécnica pública de gran tamaño.

En un contexto marcado por los cambios radicales en el método de aprendizaje de la era digital y la aparición de comunidades de aprendizaje en línea y en la vida real, el mundo universitario sigue manteniendo la hipótesis muy extendida de que ni la innovación ni la educación forman parte de la «excelencia». Y, sobre todo entre las universidades politécnicas, a menudo la innovación sigue siendo percibida como un sustituto de las soluciones impulsadas por la ciencia y la tecnología. Además, las nociones de espíritu emprendedor y emprendimiento, así como el concepto de universidad emprendedora, presentan connotaciones confusas o directamente negativas en múltiples entornos, tanto dentro como fuera del campus.

Las universidades españolas parecen bastante tradicionales y están en constante lucha para discernir cómo adaptarse a las nuevas necesidades de sus estudiantes, a los paradigmas de ciencia abierta y a las condiciones de un mercado de trabajo que demanda nuevos tipos de competencias. Su relación con la aparición de nuevos espacios de aprendizaje, que tienen una presencia cada vez mayor en el modelo educativo del Siglo XXI, parece distanciarlas aún más de la realidad no académica en constante cambio. Y la digitalización y la transformación digital representan tan solo un aspecto de este problema.

A la reunión asistieron más de 50 personas procedentes de 15 países de Europa y América del Norte. Para favorecer la reflexión, RISE y la UPC invitaron a expertos y facultativos internacionales, representantes de oficinas de transferencia de tecnología, gestores de aceleradoras universitarias, el IET y los $\mathrm{CCl}$, las administraciones públicas central y autonómica, representantes de pymes, empresas y aceleradoras privadas, así como el Centro Común de Investigación (JRC, por sus siglas en inglés) y la Comisión Europea. Estos llamaron la atención sobre la necesidad de incorporar nuevos patrones organizativos y colaboraciones público-privadas, además de nuevos modelos de negocio y de financiación para instituciones y organizaciones, que deben maximizar el impacto de los conocimientos que producen y difunden. La desventaja reside en que los cambios radicales, las disrupciones y los modelos híbridos del sector educativo, que vinculan el mundo físico con el virtual y digital y el entorno académico con el no académico, exigen nuevas políticas de arriba hacia abajo y nuevas estrategias ejecutivas de abajo hacia arriba. Si bien la disrupción puede extenderse rápidamente a lo largo y ancho de un sistema, tanto los dirigentes políticos como los responsables universitarios se enfrentan a un reto similar: cómo responder con más agilidad y flexibilidad y adoptar estos cambios en lugar de estar sujetos a la exclusión, la fuga de talento y la inercia organizativa.

Las conclusiones de las reuniones pueden resumirse de la siguiente manera:

En primer lugar, el desafío clave está relacionado con el talento. La transformación de la universidad tradicional orientada a la investigación en una institución emprendedora centrada en la innovación representa un desafío universal, no solo específico de Europa. Sin embargo, este proceso resulta cada vez más complicado para las universidades europeas, ya que están envejeciendo al tiempo que se incrementa el promedio de edad entre el profesorado. En muchos casos, la competencia para convertirse en profesor titular ha crecido y los profesionales académicos más jóvenes perciben cada vez más que su futuro depende de ellos mismos y no pasa por obtener un puesto de trabajo vitalicio.

En segundo lugar, la transformación conlleva un conflicto de prioridades en una relación aparentemente sinérgica. Así, la transformación de las universidades europeas pasa por mantener la excelencia centrada en la investigación y, al mismo tiempo, por adoptar un nuevo enfoque en torno a la innovación y el emprendimiento.

En tercer lugar, la transformación está vinculada a los cambios radicales que están alterando el mercado laboral y a la necesidad de los distintos sectores por 
incorporar nuevos tipos de graduados. La escasez de talento es citada a menudo como el obstáculo principal para el crecimiento de los líderes empresariales. Atraer a estudiantes y jóvenes investigadores a alianzas colaborativas entre industria y sociedad requiere la aplicación de cambios profundos en los currículos, los sistemas de incentivos y los patrones laborales. Resulta caro desarrollar programas de formación a medida y explorar nuevos modelos de colaboración entre el mundo empresarial, académico y social. El fortalecimiento de las actividades de transferencia de conocimiento, la comercialización de la propiedad industrial y la creación de startups son para la mayoría de las universidades actividades todavía marginales, más que una ambición estratégica.

En cuarto lugar, existe una serie de temas convergentes para las universidades emprendedoras en el contexto de la innovación abierta, que incluyen el cometido fundamental de las universidades como conectores de ecosistemas, la necesidad de realizar cambios en la gestión pública y en la cultura académica de cara a impulsar la apertura a la mentalidad emprendedora, la función crucial de la multidisciplinariedad y la interdisciplinariedad y la reformulación del papel de la propiedad intelectual en los regímenes de ciencia abierta.

La reunión se centró en la innovación en el ámbito académico y la transformación de los campus y, posteriormente, se realizaron diversas visitas a espacios de aprendizaje colaborativo: Barcelona Activa y Pier 01 Barcelona Tech City. Un interesante resultado de estas reuniones fue la conclusión de que ciudades europeas han empezado a tener bastante éxito en la adopción de estos cambios multidimensionales, que las universidades como tales se esfuerzan por realizar. Barcelona, que como ciudad representa uno los ejemplos globales más reconocidos de transformación urbana a gran escala, sirvió de ejemplo práctico inspirador. Su proyecto 22@Barcelona ha permitido convertir los polígonos posindustriales de PobleNou en uno de los entornos innovadores más vibrantes del mundo, ya que atrae talento multicultural, empresas multinacionales $\mathrm{y}$ capital riesgo. La inmersión en el ecosistema innovador del área metropolitana de Barcelona ha brindado conocimiento bastante novedoso sobre los modelos de crecimiento que necesita España.

\section{Políticas orientadas a misiones. Un caso práctico: misión de EIT Alumni «Reinvent Badalona»}

En el informe Horizonte Europa, recientemente publicado, se establece que las políticas orientadas a misiones concretas constituyen un nuevo método para que las I+i logren un impacto más profundo. Se espera que el cambio de grandes desafíos a misiones concretas marque el rumbo, incremente el ritmo y organice a los socios a fin de recorrer el camino hacia la consecución de un objetivo tangible común, oportuno y centrado en los resultados más que en la creación de capacidades. Las preguntas sobre cómo se desarrollarán las misiones, de qué tipo serán y cómo involucrar a los ciudadanos en el eficaz fomento de las mismas para tender puentes entre «el poder y el significado» continúan siendo objeto de análisis.

Este es un claro ejemplo de cómo las políticas de $\mathrm{I}+\mathrm{i}$ orientadas a misiones concretas podrían conjugarse con la política de desarrollo regional para generar un impulso a gran escala en toda Europa. A gran escala, dado que está relacionado con el modelo de crecimiento de las ciudades de mediano tamaño que, a menudo, están a la sombra de las grandes metrópolis de sus regiones; estos municipios sufren la fuga de cerebros y no logran atraer los recursos clave para fomentar la innovación: el talento, la financiación y el conocimiento.

En marzo de 2018, el EIT Alumni (antiguos alumnos del IET) planteó a Tataj Innovation la idea de patrocinar sus Startup Days. Tataj Innovation es una empresa europea de estrategia e inversión que ayuda a que empresas de mediano tamaño construyan distritos de la innovación. EIT Alumni es una federación compuesta por las asociaciones de antiguos alumnos de las comunidades de conocimiento e innovación del IET: Climate 
KIC, KIC Innoenergy, EIT Digital y EIT Health. Con el apoyo de las oficinas del IET en Budapest, organizan hackathons en toda Europa para consolidar la comunidad del EIT Alumni que, en la actualidad, incluye en su seno más de 4.000 licenciados con espíritu empresarial de las mejores universidades de investigación del continente. Estos son algunos de los mejores talentos que Europa tiene en la actualidad con capacidad disruptiva para acelerar el crecimiento de la UE. Esta capacidad se basa en tres aspectos:

- el ADN de los graduados que están interesados en la creación de empresas, que gozan de las habilidades para crear startups nacidas con alcance global y gestionar su crecimiento para convertirse en scaleups y que estudiaron en los programas educativos internacionales de los $\mathrm{CCl}$ del IET centrados en aportar competencias empresariales a la I+i;

- pertenecer a las comunidades CCI del IET a nivel europeo como entorno de innovación abierta de confianza que reúne a algunas de las principales empresas globales y locales en un sistema de innovación dinámico estructurado; ser parte de esta comunidad acorta el tiempo que transcurre hasta que los resultados de las investigaciones llegan al mercado, brindando acceso más directo a la creación conjunta de innovación con usuarios y clientes y a sus cadenas de valor globales;

- el efecto de red, que supone el elemento nuclear del modelo de gobernanza del IET, diseñado para generar un crecimiento exponencial en Europa.

«Reinvent Badalona» era la misión que Tataj Innovation encomendó a los participantes de los Startup Days de EIT Alumni en Barcelona en marzo de 2018. Badalona es una ciudad de 200.000 habitantes situada en la zona metropolitana de Barcelona, compuesta por la propia Barcelona y multitud de ciudades de tamaño mediano y pequeño que se han fusionado con la expansión de la economía local. En una época esta área era conocida como el «Mánchester catalán» $y$, de hecho, era la segunda zona metropolitana más industrializada del mundo. Tras luchar durante décadas, Barcelona se embarcó en un nuevo plan de desarrollo urbano destinado a prepararse para la celebración de las Olimpiadas de 1992. Asimismo, en 2014 ganó el título de Capital Europea de la Innovación en la primera competición organizada por la Comisión Europea. Hoy, Barcelona es la cuarta ciudad de Europa en materia de startups y una de las mejores ciudades en calidad de vida del mundo. Más del 40 por 100 del total de la inversión de capital riesgo en España va destinado a empresas sitas en Cataluña. El principal proyecto de desarrollo urbano, 22@Barcelona, se ha convertido en un ejemplo de referencia global para los responsables de la formulación de políticas urbanas.

El éxito de Barcelona ha tenido un efecto mixto en las ciudades adscritas a su área metropolitana, como Badalona. Por un lado, ha creado oportunidades de empleo para los ciudadanos que se desplazan diariamente a la ciudad de Barcelona. Por otro, la propia Barcelona concentra a inversores, universidades y empresas. 22@Barcelona y el puerto de Badalona se encuentran a una distancia de diez minutos en coche y separadas por una vasta área de 80 hectáreas de polígonos posindustriales (espacios urbanos deteriorados con antiguas fábricas y almacenes). El desafío —y la oportunidadradica en cómo acelerar la transformación de esta vieja gloria del pasado industrial en la industria 4.0. Cómo crear la «costa del conocimiento» que incluya toda la región metropolitana de Barcelona, desde Sitges a Mataró.

\section{Cómo crear rápidamente un prototipo de distrito de la innovación: el modelo de Badalona}

Aún no se ha demostrado si los Startup Days de EIT Alumni acelerarán la transformación urbana de Badalona. Las evidencias empíricas mostrarán si la ciudad puede atraer la inversión necesaria para incubar un distrito de la innovación. Adoptando una perspectiva más amplia como evidencia, basar el modelo de crecimiento del Network Thinking en la experiencia del establecimiento del propio IET y las comunidades de conocimiento e innovación podría resultar de gran utilidad para rediseñar e implantar nuevas políticas y 
estrategias de crecimiento para ciudades posindustriales de mediano tamaño como Badalona.

EI EIT Alumni ha propuesto cinco ideas que podrían servir de inspiración de cara a la puesta en marcha de nuevos proyectos emblemáticos para Badalona, San Adrián de Besós o Montgat. En principio, la restauración del paseo marítimo entre Pont del Petroli y el puerto de Badalona ha sido concebida como una extensión de22@Barcelona y el plan urbanístico está en la actualidad en fase de elaboración. La misión del proyecto «Reinvent Badalona» del EIT Alumni incluye:

- Puente a China: reimaginar el puerto de Badalona y construir un centro cultural y empresarial para realizar actividades comerciales con Asia, ya que Badalona acoge la mayor concentración de empresas chinas de España, con unas 600 entidades.

- BADALONA 4.0: crear el Artificial Intelligence Living Lab como núcleo centralizado para experimentar las tecnologías de la IA y crear un espacio multifuncional para la formación y las redes de trabajo.

- eNEUTRAL: construir un oasis respetuoso con el medioambiente como distrito energéticamente neutral, que combina el uso público, comercial y residencial del suelo. Elaborar un modelo para nuevos modos de vivir, viviendas sin huella de carbono, invernaderos y huertos urbanos, todos ellos conectados a través de soluciones de movilidad sostenible.

- Pico Blau, el paraíso del surf: desplegar tecnologías de eficacia contrastada para crear un arrecife artificial para surfistas $y$ aprovechar el potencial de las playas $y$ la tradición deportiva de Badalona. La ciudad cuenta con uno de los litorales más extensos del mar Mediterráneo y la reconocida trayectoria del Club Natació Badalona es una buena base para crear nuevas disciplinas deportivas y promover un estilo de vida sano y equilibrado.

- Open Social Lab: convertir la central eléctrica de ENDESA, con sus tres icónicas chimeneas, en un centro clave para la actividad social y la innovación con un 3D Print Hub y un Makers' Space destinado a emprendedores, innovadores, artistas, diseñadores y visionarios de todo el mundo.
La aplicación de la metodología del Network Thinking para transformar ciudades de tamaño medio como Badalona representa en sí misma una innovación y es un proceso de aprendizaje que debe gestionarse adecuadamente y validarse. El 21 de junio de 2018, en la sala comunitaria del puerto de Badalona, más de 40 representantes del ecosistema local se reunieron para una clase magistral en innovación sobre Network Thinking a cargo del profesor Manuel Castells y la doctora Daria Tataj, autora del presente artículo. La clase se centró en explicar una vez más que el modelo de Silicon Valley no se puede replicar y que cada ciudad, grande o pequeña, necesita reinventar su propio modelo de crecimiento en función de los principios que rigen la sociedad en red y los cuatro pilares fundamentales de cualquier economía del conocimiento: investigación, educación, innovación y espíritu emprendedor.

El siguiente elemento de este proceso que valida la posibilidad de crear prototipos rápidos de distritos de la innovación es el Innovation Retreat del EIT Alumni en Tataj Innovation Studio Badalona Beach Barcelona, un nuevo laboratorio creativo para la incipiente comunidad de innovadores de la ciudad. Durante el retiro, los participantes del hackathon celebrado en marzo se volverán a reunir para seguir el proceso de formación en Network Thinking y participarán en un ejercicio estratégico sobre cómo crear y captar valor y cómo implicar al ecosistema local y global para impulsar dicho valor.

Es posible que alguno de estos cinco proyectos pueda convertirse en una verdadera propuesta de valor para la inversión. Y la transformación urbana siempre crea nuevas oportunidades empresariales. La pregunta que debemos formularnos es si el gobierno local y las principales empresas se comprometerán a crear e invertir conjuntamente en el desarrollo de estos proyectos. ¿Podrán los agentes locales colaborar con otra ciudad europea, por ejemplo la de Lodz en Polonia, para crear una alianza transregional y desarrollar conjuntamente una perspectiva sobre cómo combinar los fondos de desarrollo e innovación regionales, en un esfuerzo pionero por demostrar que las ciudades de mediano 
tamaño pueden acelerar la aparición de distritos de la innovación vibrantes, estrechamente unidos a nivel local y que mantengan una relación de estructura flexible a escala global? El modelo de crecimiento Network Thinking es relevante en el contexto de la economía de redes y en un futuro cercano tendremos los resultados de este proceso de aprendizaje mediante la práctica, que puede tener un efecto transformador en Badalona y en otras ciudades similares.

\section{Conclusiones}

El argumento esgrimido en este artículo sugiere la necesidad de apuntalar una transformación sistémica en el núcleo de la renovación de la economía española, que incluye reformas estructurales y un cambio cultural hacia el emprendimiento, no solo en las principales áreas metropolitanas como Madrid o Barcelona, sino también en muchas otras ciudades de tamaño medio, que pueden convertir sus zonas posindustriales en vibrantes distritos de la innovación.

La destrucción creativa, esto es, la esencia última del ADN emprendedor que renueva el impulso y reinventa la libertad de la economía de mercado motivada por la «mano invisible», elimina paulatinamente los modelos obsoletos e ineficientes de las prácticas empresariales. No tendrá piedad de aquellas empresas españolas que no aprendan a innovar de forma más eficaz y rápida. Este proceso de aprendizaje no se produce por sí solo, sino que necesita un ecosistema que incluya a instituciones educativas, Administraciones Públicas y la sociedad en su conjunto. Las empresas que no logren adaptarse con suficiente rapidez, reinventarse a sí mismas y adoptar el cambio están condenadas al fracaso, dejando margen y nichos de mercado libres que otras podrán aprovechar.

Los modelos emergentes de creación de valor en la economía de redes, concebidos en el marco del Network Thinking mencionado anteriormente, han surgido de la investigación y de las prácticas destinadas a sacar partido de las dinámicas de los flujos de conocimiento, talento y capital en la red de redes de la innovación global. Este paradigma del Network Thinking se ha diseñado para guiar, ayudar y, en última instancia, crear conjuntamente nuevas formas de administrar y organizar las redes de la innovación. Todo con el fin de fomentar la creación y la captura de valor.

Al final, los principios subyacentes del modelo de crecimiento para España deben basarse, por un lado, en los fundamentos del Network Thinking y, por otro, en los cuatro pilares de una economía de la innovación: la investigación, la educación, la innovación y el espíritu emprendedor. Estos pilares pueden convertirse en la base del crecimiento únicamente si se interconectan y se integran en un sistema de innovación a través del paradigma del Network Thinking. Después de todo, es una cuestión de identidad. La identidad de los lugares y la identidad de las personas. Con respecto a los lugares, o más bien los nodos de la red de innovación global, las preguntas que debemos plantearnos son las siguientes: ¿lograrán las ciudades españolas de tamaño grande y mediano atraer y mantener el talento, el conocimiento y la financiación? ¿podrán reinventar su herencia industrial y transformar los polígonos posindustriales en distritos de la innovación? Y, ¿podrá la generación de jóvenes españoles titulados y los profesionales a mitad de carrera -empujados por la necesidad o por la exigencia creativa- llevar a cabo y ampliar los proyectos empresariales? Y en tal caso, ¿podrán estos emprendedores innovar mejor y más rápido abrazando la capacidad de España en términos de investigación, educación e innovación y transformando dicha capacidad en un sistema que genere crecimiento y puestos de trabajo en el país, o en otros lugares?

\section{Referencias bibliográficas}

[1] BANCO MUNDIAL (2018). Lessons from Poland, Insights for Poland. http://www.worldbank.org/en/country/poland/publication/lessons-from-poland-insights-for-poland

[2] CARACA, J.; LAGO, T. y TATAJ, D. (2017). «European Science and Technology in a Time of Crisis: ERC, EIT and Beyond», en CASTELLS, M. (ed.), La crisis de Europa, Polity Press. 
[3] CASTELLS, M. (2017). Ruptura. La crisis de la democracia liberal, Alianza Editorial.

[4] GEORGHIU, L. et al. (2018). Mission-oriented Research and Innovation Policy, A Study by RISE, Dirección General de Investigación e Innovación. Comisión Europea. https://publications.europa.eu/en/publication-detail/-/publicati on/61e742ed-2358-11e8-ac73-01aa75ed71a1/language-en

[5] MAZZUCATO, M. (2018). Mission-oriented Research and Innovation in the European Union, Dirección General de Investigación e Innovación, Comisión Europea. https://ec.europa.eu/info/sites/info/files/mazzucato_report_2018.pdf

[6] REAL. E. y TATAJ, D. (2018). Universitats emprenedores al segle 21. Estratègies, incentius i polítiques de finançament que permetin integrar els processos d'innovació oberta i collaborativa amb l'ecosistema local i global. 3r Congrés d'Economia i Empresa de Catalunya; $h$ ttps://www.scipedia.com/public/Real_2018a

[7] TATAJ, D. (2014). Poland. An Emerging Innovation Leader of the Vysegrad Group, Ministerio de Relaciones Exteriores de Polonia, Varsovia. https://ec.europa.eu/research/openvision/index.cfm
[8] TATAJ, D. (2015). Innovation and Entrepreneurship. A Growth Model for Europe beyond the Crisis, prefacio por Manuel Castells, Tataj Biblioteca de Innovación, Nueva York.

[9] TATAJ, D. (2108). «Utopías necesarias. Economía intangible» (audiovisual), Informe Cotec 2018. http://informecotec.es

[10] TATAJ, D.; VERGANTI, R. et al. (2017). «The European Innovation Council: Strategic Reflection», en SOETE, L. (ed.) Europe's Future: Open Science, Open Innovation, Open to the World, Grupo RISE de expertos de alto nivel para el High-level Expert Group, Comisario de la UE, Dirección General de Investigación e Innovación, Comisión Europea. https://publications.europa.eu/en/publication-detail/-/ publication/527ea7ce-36fc-11e7-a08e-01aa75ed71a1

[11] VON GABAIN, A. y TATAJ, D. (2014). The EIT's Knowledge and Innovation Communities (KICs): Towards European Innovation Factories. Open Innovation Yearbook 2014, Comisión Europea. https://ec.europa.eu/digital-single-market/en/news/ open-innovation-20-yearbook-2014-giving-you-stimulus-andideas 